\title{
Gestión de la calidad educativa y rendimiento académico en la institución educativa Arequipa
}

\section{Educational quality management and academic performance in the Arequipa School}

Walter L. Arias Gallegos', Silvia F. Zavala Delgado², Alberto Bernilla Meneses ${ }^{3}$ Universidad Católica San Pablo, Perú

\section{RESUMEN}

Objetivos: Analizar la relación que existe entre la percepción de la calidad de la gestión educativa y el rendimiento académico de un grupo de estudiantes de la Institución Educativa Arequipa con edades comprendidas entre los 12 y 17 años y desde primero hasta quinto de secundaria. Métodos: La muestra consta de 110 escolares mujeres que han sido seleccionadas mediante técnicas de muestreo probabilístico. Se aplicó el cuestionario de percepción de la calidad educativa, que consta de 17 ítems y cuenta con adecuados índices de validez y confiabilidad $(\alpha=0,848)$. Para el procesamiento de los datos se han utilizado estadísticos descriptivos (media, desviación estándar, rango y varianza), además de pruebas paramétricas de correlación (Coeficiente de Pearson) para las variables cuantitativas y pruebas no paramétricas (Prueba Tau-b de Kendall) para las variables cualitativas. Resultados: La puntuación promedio de percepción de la calidad global fue de 50,29 con una desviación estándar de 9,14 y un rango de 22 a 65 . No existen relaciones significativas entre las variables de estudio para el total de la muestra, pero sí cuando los datos se procesan en función del grado de escolaridad, sobre todo en primero $(r=0,37)$ y cuarto de secundaria $(r=0,36)$. Conclusiones: Se concluye que sí existe relación entre el rendimiento académico y la percepción de la calidad educativa, pero ésta es diferenciada, según el grado de estudios.

Palabras clave: Rendimiento académico, percepción, calidad educativa, gestión.

1 Psicólogo; Maestro en Ciencias de la Educación con mención en Psicopedagogía Cognitiva de la Universidad Nacional Pedro Ruíz Gallo; Docente investigador de la Universidad Católica San Pablo.

2 Profesora en Educación Secundaria; egresada de la maestría en Ciencias de la Educación con mención en Gestión de la Educación de la Universidad Católica San Pablo; Docente de la I. E. "Arequipa".

3 Estudiante del Programa Profesional de Educación de la Universidad Católica San Pablo. 


\section{ABSTRACT}

Objectives: To analyze the relationship between the educational quality management perception and the academic performance in a group of 12-17 year old School Arequipa's students from first to fifth grade. Methods: The sample consisted of 110 women students, they have been selected by probability sampling techniques. Educational quality perception Questionnaire was applied, which consists of 17 items and has adequate validity indices and reliability $(\alpha=0,848)$. For data processing, descriptive statistics (mean, standard deviation, range and variance) were used, also correlation parametric tests (Pearson coefficient) for quantitative variables and nonparametric tests (Kendall's Tau-b Test) for qualitative variables. Results: The average score of global quality perception was 50,29 with a standard deviation of 9,14 and a range of 22 to 65 . There are no significant relationships between the study variables for the total sample, but there is significant relationship when data is processed according to the education level, especially in first grade $(r=0,37)$ and fourth grade $(r=0,36)$. Conclusions: We conclude that there is a relationship between academic performance and educational quality perception, but it is differentiated by student grade.

Keywords: Academic performance, perception, educational quality, management.

\section{INTRODUCCIÓN}

La gestión de la calidad educativa es un enfoque reciente, que ha sido adaptado al ámbito educativo desde el concepto de calidad total que se aplica en las empresas y los sistemas de producción de bienes (1). Puede decirse que surge en la era preindustrial, en el silgo XIII, cuando empieza a predominar una visión orientada a los negocios; mientras que en la era industrial, los esfuerzos de Taylor y de Fayol representan la antesala de la formalización de los sistemas de calidad. En la segunda década del siglo $X X$, el estudio de Elton Mayo puso énfasis en el capital humano y contribuyó con el desarrollo de un enfoque centrado en el trabajador, que es el núcleo de los modelos de calidad (2). En 1924, Shewhart introduce el control estadístico de la calidad, en tanto que Deming en 1950 y en 1953 Ishikawa, hicieron una contribución más significativa al plantear, el modelo de la calidad total (3).

En palabras de Salazar y García (3) la calidad es un atributo que distingue las cosas, las personas, los bienes y los servicios. La calidad de un producto o un servicio define entonces, las propiedades y las características que le confiere su aptitud - capacidad para satisfacer necesidades explícitas o implícitas. Capella (4) define a calidad de la educación como la pertinencia de los sistemas educativos para responder las demandas socioculturales, ya que las escuelas son transmisoras de pautas culturales y de escenarios para la socialización. Para nosotros la calidad es el atributo de una cosa, objeto, servicio o hasta una persona; que designa su competencia, eficacia y eficiencia en función del fin al que se debe.

Aunque el concepto de calidad ha sido aplicado principalmente en el mundo de las empresas y la producción industrial o manufacturera, en educación el concepto de calidad reviste de vital importancia. En ese sentido, cuando la calidad se aplica a los servicios, más que a los bienes, hay quienes prefieren usar el término de gestión de la calidad, que resulta muy conveniente para el campo de la educación.

En consecuencia, la gestión educativa es una disciplina de la gestión en la que interactúan la teoría, la política y la pragmática. Los ámbitos de la gestión educativa son la gestión institucional, la gestión pedagógica y la gestión administrativa (5). A nivel institucional, la promoción de una educación de calidad se expresa en la generación de proyectos de desarrollo institucional que respondan a las necesidades de la comunidad y de los estudiantes (6), ya que la educación debe 
estimular la comprensión del propio medio y la solución de problemas sociales (5).

El director es quien por poseer el poder formal en la institución educativa, es el responsable de la calidad institucional, que abarca la calidad pedagógica y también la calidad administrativa. El director consigue esto a través de sus funciones de planificación, ejecución, coordinación y supervisión del servicio educativo (7). De modo que cuando en la institución se planifica, se guía la organización hacia el logro de sus fines y objetivos (8).

Así pues, son los directores, quienes animando a los docentes que integran la comunidad educativa deben generar proyectos de desarrollo (9), planes innovadores y proyectos educativos (10) que mitiguen las limitaciones del sistema y por el contrario maximicen las potencialidades y los recursos con que se cuenta. En ese sentido, el perfil del director y el rol del profesor han cambiado drásticamente desde los 90'. El primero es ahora un facilitador del aprendizaje y el segundo es un gestor de la calidad educativa (11).

Ahora bien, la gestión de la calidad educativa conlleva cambio. En los 40' los procesos de cambio se basaban en modelos causa-efecto, mientras que en los $50^{\prime}$ surgieron los modelos de viabilidad de cambio, y hoy en día, predominan los modelos de complejidad. El cambio es un concepto nodal de la gestión, ya que la calidad constituye una revolución en el pensamiento, pues implica crear nuevas formas de organizar el sistema educativo y repensar el proceso de aprendizaje (4). Por ello, el aprendizaje organizacional es la clave de la gestión y el desarrollo de las instituciones educativas.

Implementar cambios empero, no es fácil, ya que como organización, las escuelas asocian un conjunto de estructuras que las definen como tales: se tienen las normas, el organigrama, el plantel, los trabajadores, etc. La gestión educativa abarca todos estos elementos y muchos más; por esta razón, la calidad educativa es un constructo multidimensional (12). Esto supone que la gestión de la calidad debe ser global, pues abarca a las personas, a los procesos, a la evaluación, a los resultados, etc. (4). Desde un enfoque sistemático, el cambio institucional comprende cuatro fases: la iniciación, la implementación, la institucionalización y la sostenibilidad; siendo ésta última la que representa mayores problemas, debido a la falta de órganos de apoyo que respalden el cambio (13).

Por ello, para gestionar el cambio educativo, se debe asumir un modelo de gestión educativa, reconociendo la escuela como organización compleja, con nuevos roles y nuevas necesidades (4). Hoy pesa más el capital intelectual que el capital económico. El capital intelectual está constituido por el toda la información y el bagaje personal que poseen los individuos (2). Por ello, una organización inteligente debe desarrollar la calidad de juicio, el dominio personal, la gestión de modelos mentales y el trabajo en equipo. Competencias de incalculable valor, si se considera que las organizaciones del futuro serán aquellas que tengan capacidad de aprender haciendo uso de su capital intelectual (14).

Dado que la calidad se manifiesta en todas las instancias de la vida educativa, como su estructura, el sistema educativo, la organización escolar, la práctica pedagógica, etc. (15), podemos ubicar la calidad en lo macrosocial como en lo microsocial. En el primer caso, tenemos el sistema educativo con el respectivo plan educativo nacional y el diseño curricular, etc.; mientras que en el segundo caso, hablamos del contexto escolar, el aula, y hasta el binomio profesor-alumno (16).

A este nivel, el de la escuela, la gestión educativa se divide en administrativa y pedagógica (4), con la salvedad que lo administrativo abarca la dirección de la escuela, donde se formulan diversas iniciativas orientadas para la mejora de las instituciones educativas. Lamentablemente, muy pocas prosperan, quizá las menos trascendentes. En parte porque estas 
propuestas no garantizan la calidad de los sistemas escolaressino haylas oportunidades materiales y técnicas necesarias para alcanzarlos (17). En ese sentido, entre los elementos adversos que dificultan la gestión de la calidad educativa se tienen la falta de motivación, la desactualización profesional, el pensamiento rígido, la dificultad de integración social en grupos de docentes, las relaciones humanas insatisfactorias, etc. (4).

Todos ellos, recaen sobre la persona, por ello Álvarez (2) señala que nada cambia sin transformación personal. El aprendizaje organizacional, es por tanto, un factor decisivo para la gestión de la calidad educativa, porque el aprendizaje organizacional es un proceso en el que una organización obtiene y realiza nuevos conocimientos, destrezas, conductas y valores. El aprendizaje organizacional enfatiza la creación de saberes apuntando hacia el desarrollo de capacidades cognitivas y la adquisición de estrategias. Toda organización que adopta un enfoque de aprendizaje organizacional se caracterizara por considerar al individuo como el centro de la organización y se preocupa por el desarrollo constante de sus capacidades organizacionales.

Las capacidades organizacionales constituyen todas las fuerzas colectivas que atraviesan el escenario educativo e integran aspectos tanto estructurales como culturales (13). Entre las capacidades de cambio y por ende, para la gestión educativa tenemos la capacidad de autoevaluación (que predice el desarrollo organizacional), la gobernabilidad (que reposa en la visión estratégica), la capacidad de regulación interna (que abarca la evaluación y la información), el trabajo en equipo (sobre la base de las relaciones humanas), el compromiso colectivo (que pare de una visión compartida por la comunidad educativa) y la atmosfera de la organización escolar (que integra todas las anteriores) (2).

Ahora bien, una manera de ver si la institución escolar cumple con las exigencias de la sociedad y qué tanto desarrolla un servicio de calidad es a través de la evaluación institucional (8). La evaluación es un proceso complejo que tiene como punto de partida el recojo de la información que puede ser de varias maneras (18). La evaluación, dentro del marco de la gestión de la calidad educativa es un elemento primordial para el perfeccionamiento de las organizaciones. Todos los modelos de evaluación que se centran en la mejora toman como piedra angular la evaluación (8). Sin embargo, dentro de este enfoque la evaluación no adquiere un matiz punitivo, sino simplemente, de retroalimentación.

Los profesores, otra pieza clave de la gestión de la calidad educativa, se oponen a la evaluación, lo que dice ya mucho de su compromiso con el cambio. Por ejemplo, de los 260000 maestros que existen en el Perú, solo el 10 por ciento se ha acogido a la Ley de la Carrea Pública Magisterial (19), precisamente, porque consideran que la evaluación es una estrategia política que pone en riesgo su permanencia en el sistema educativo estatal.

La evaluación docente empero, es una necesidad ineludible, dados los bajos estándares de la calidad educativa, que de acuerdo con la pruebas PISA el $75 \%$ de los estudiantes del Perú no comprende lo que lee (19). Estos datos ponen en tela de juicio la calidad de los aprendizajes de los estudiantes y en consecuencia, también la calidad de la enseñanza. En ese sentido, la evaluación de la calidad se ha centrado en el desempeño académico, más que con el tipo de gestión de las escuelas, ya sean públicas o privadas (15).

Así pues, algunos estudios han planteado que la calidad de la gestión educativa, en sus diversos niveles puede relacionarse con el rendimiento académico de los estudiantes. Los resultados y los métodos para estudiar estas relaciones han sido muy variados. En un estudio cualitativo por ejemplo, se encontró que los docentes perciben que el director ponía mucho énfasis en el control administrativo y que bloqueaba las iniciativas pedagógicas de 
los profesores, mientras que los estudiantes le percibían como un amigo (7).

Otro estudio realizado en el ámbito de la educación superior, buscaba hallar relaciones entre el rendimiento académico de los estudiantes universitarios y la percepción que tienen de la calidad académica de sus profesores, sin embargo, no se encontraron relaciones significativas entre ambas variables (20).

Así pues, el cómo los estudiantes se perciben a sí mismos, a sus profesores, a su colegio e incluso al sistema escolar más próximo; puede afectar su conducta y su aprendizaje. Un estudiante que perciba que su entorno educativo es negativo, y que sus posibilidades de sobresalir son escasas, seguramente invertirá menos esfuerzos en sus logros académicos. En cambio, un estudiante que percibe que tiene buenas posibilidades de éxito, seguramente tendrá mayor motivación para estudiar.

Por ello, no puede descuidarse la gestión de la calidad educativa, ya que la manera en que perciben los estudiantes las diversas esferas educativas, tales como el dirección, la docencia y la administración de los servicios educativos; pueden tener un impacto en su aprendizaje y por ende, también en su rendimiento académico

Con base en todo lo dicho, en el presente estudio se pretende determinar el grado de relación entre la percepción de la gestión de la calidad educativa que tienen las estudiantes del nivel secundario de la I.E. Arequipa, con su rendimiento académico. Partimos de la hipótesis de que entre las variables de estudio hay relaciones positivas y significativas, así como entre cada uno de sus componentes.

\section{MATERIAL Y MÉTODOS}

El tipo de investigación de este estudio es cuantitativo con un diseño de investigación correlacional (21).

La población para efectos de la presente
Tabla $N^{\circ} 1$ : Composición de la muestra

\begin{tabular}{lrr}
\hline \multicolumn{1}{c}{ Grado } & Población & Muestra \\
\hline Primero & 176 & 22 \\
Segundo & 141 & 20 \\
Tercero & 180 & 23 \\
Cuarto & 201 & 22 \\
Quinto & 226 & 23 \\
\hline Total & 924 & 110 \\
\hline
\end{tabular}

investigación se encuentra compuesta por estudiantes entre 12 y 17 años (aproximadamente) del nivel secundario, de la I. E. Arequipa, que suman la cantidad de 924. El tamaño de muestra es de 110 estudiantes de nivel secundario de la I. E. Arequipa, como se aprecia en la tabla 1.

Respecto a los instrumentos para la recolección de datos de la variable independiente variable dependiente, se hizo uso de un cuestionario estructurado que se ha diseñado para los fines específicos de la investigación. El cuestionario comprende preguntas cerradas y con alternativas de respuesta tipo Likert que se puntúan de 4 (para siempre) a 0 (para nunca), bajo la denominación de Cuestionario de percepción de la calidad en la escuela.

El cuestionario comprende cuatro partes. La primera es para los datos personales como edad, sexo, grado de estudios, sección y la nota promedio obtenida en el último bimestre evaluado previo al recojo de la información.

La segunda parte se refiere a la percepción de la dirección y se compone de siete preguntas destinadas a valorar la calidad del desempeño de la dirección en función de los indicadores de liderazgo, gestión de proyectos educativos, organización y control. La tercera parte se relaciona con la percepción de la docencia con seis ítems sobre el conocimiento del profesor, la didáctica empleada, la evaluación, su responsabilidad y su compromiso con el estudiante.

Finalmente la cuarta parte recoge datos de la percepción de las estudiantes acerca de la calidad de la administración en la escuela, 
Tabla N ${ }^{\circ}$ 2: Valores descriptivos de las variables de estudio.

\begin{tabular}{lrrrrrr}
\hline Var. y dimens. & Media & Desy Est. & Mínimo & Máximo & Rango & Varianza \\
\hline Nota promedio & 12,9 & 1,82 & 10 & 20 & 10 & 3,34 \\
P. de calidad en dirección & 19,5 & 4,32 & 7 & 26 & 19 & 18,69 \\
P. de calidad docencia & 20,1 & 3,06 & 12 & 24 & 12 & 9,41 \\
P. de calidad administrat. & 10,6 & 3,12 & 0 & 15 & 15 & 9,77 \\
P. global de la calidad & 50,2 & 9,14 & 22 & 65 & 43 & 83,60 \\
\hline
\end{tabular}

con preguntas dirigidas a la atención, la realización de trámites y la solución de problemas.

Para determinar la validez del instrumento se hicieron correlaciones ítem-test con la finalidad de valorar la homogeneidad de contraste de cada uno de los ítems. El rango de las correlaciones obtenidas va de 0,314 a 0,743 según el coeficiente de Pearson, por tanto se puede decir que los ítems miden un mismo constructo como es la percepción de la calidad educativa.

Con respecto a la confiabilidad del instrumento se obtuvieron cuatro medidas de consistencia interna a través de la prueba Alpha de Cronbach. En los cuatro casos se obtuvieron índices aceptables, siendo muy altos en los ítems que miden la percepción de la calidad en el director $(\alpha=0,828)$, la percepción de la calidad de los docentes $(\alpha=0,871)$ y en la percepción de la calidad como valor total, donde se obtuvo un valor Alpha de Cronbach de $\alpha=0,848$. El menor valor se registró en percepción de la calidad en administrativos $(\alpha=0,651)$. Ya que en todos los casos se supera el índice de 0,60, se puede concluir que el instrumento es confiable, así como válido.

Los procedimientos utilizados fueron los siguientes: se recogieron los datos en horario de clases con los permisos previos correspondientes de parte de las autoridades de la I.E. Arequipa. La recolección de datos se hizo de manera colectiva una vez que se realizó la selección de la muestra, sólo las estudiantes escogidas como parte de la muestra llenaron el instrumento diseñado. Las notas del promedio fueron obtenidas de los registros académicos con la autorización de dirección. Los datos se llenaron en la ficha de registro que acompaña el Cuestionario de percepción de la calidad en la escuela. Una vez recogida la información se codificaron los datos obtenidos y se aplicó estadística descriptiva e inferencial.

\section{RESULTADOS}

Para el procesamiento de los datos se han utilizado estadísticos descriptivos (media, desviación estándar, rango y varianza), además de pruebas paramétricas de correlación (Coeficiente de Pearson) para las variables cuantitativas y pruebas no paramétricas (Prueba Tau-b de Kendall) para las variables cualitativas, según su nivel de medición (ordinal y de intervalo), mediante el programa SPSS 16.0.

Las correlaciones entre el rendimiento académico y la percepción de la calidad de las estudiantes de manera global, y luego por grado. Los valores con un asterisco $\left({ }^{*}\right)$ indican un nivel de confianza de $p<0,05 y$ los valores con dos asteriscos $\left({ }^{* *}\right)$ indican un nivel de confianza de $p<0,01$.

La nota promedio es de 12,99 con una desviación estándar de 1,82 y un rango de 10 a 20 . La puntuación promedio de

Tabla $N^{\circ}$ 3: Distribución de porcentajes por nota promedio

\begin{tabular}{cccc}
\hline Nota & Frecuencia & Porcentaje & $\begin{array}{c}\text { Porcentaje } \\
\text { acumulado }\end{array}$ \\
\hline 10 & 6 & 5,45 & 5,45 \\
11 & 13 & 11,81 & 17,27 \\
12 & 26 & 23,63 & 40,90 \\
13 & 30 & 27,27 & 68,18 \\
14 & 18 & 16,36 & 84,54 \\
15 & 10 & 9,09 & 93,63 \\
16 & 3 & 2,72 & 96,36 \\
17 & 3 & 2,72 & 99,09 \\
20 & 1 & 0,90 & 100,00 \\
\hline
\end{tabular}


percepción de la calidad en dirección fue de 19,5 con una desviación estándar de 4,32 y un rango de 7 a 26 . La puntuación promedio de percepción de la calidad en docentes fue de 20,10 con una desviación estándar de 3,06 y un rango de 12 a 24 . La puntuación promedio de la percepción de la calidad en administrativos fue de 10,68 con una desviación estándar de 3,12 y un rango de $\mathrm{O}$ a 15 . Finalmente, la puntuación promedio de percepción de la calidad global fue de 50,29 con una desviación estándar de 9,14 y un rango de 22 a 65.

En la tabla 3 se aprecia la distribución de frecuencias y porcentajes por nota promedio durante el primer bimestre de las alumnas de la I. E. Arequipa. Se puede observar que el mayor porcentaje se ubica en la nota 13
$(27,27 \%)$ y el menor en la nota $20,0,90$ $\%)$. El 23,63 \% tuvo nota promedio 12, el $16,36 \%$ tuvo 14 , el $11,81 \%$ tuvo 11 , el $9,09 \%$ tuvo 15 , el $5,45 \%$ tuvo 10 y el 2,72 $\%$ tuvo 16 y 17 , en cantidades iguales. Esto sugiere que aproximadamente, el $94 \%$ de la muestra tiene una nota probatoria y el resto ha desaprobado. Además, solo el $7 \%$ de la muestra se ubica en el quinto superior, mientras que el grueso de estudiantes tiene notas entre 11 y 15 .

En la tabla 4 muestra cómo las correlaciones entre la percepción de la calidad de la gestión en dirección, la percepción de la calidad docente, la percepción de la calidad en administrativos y la calidad total, están fuertemente relacionadas entre sí, lo cual da buena cuenta de las propiedades

Tabla $N^{\circ}$ 4: Correlaciones entre rendimiento académico y percepción de la calidad en el total de la muestra.

\begin{tabular}{lcccccc}
\hline Nota & $\mathbf{1}$ & $\mathbf{2}$ & $\mathbf{3}$ & $\mathbf{4}$ & $\mathbf{5}$ & $\mathbf{6}$ \\
\hline 1. Nota promedio & 1,00 & $-0,14$ & $-0,16$ & $-0,13$ & $-0,17$ & $-0,06$ \\
2. Calidad dirección & & 1,00 & $0,67^{* *}$ & $0,62^{* *}$ & $-0,91^{* *}$ & $-0,09$ \\
3. Calidad docencia & & & 1,00 & $0,56^{* *}$ & $0,85^{* *}$ & $-0,09$ \\
4. Calidad administrativa & & & & 1,00 & $0,82^{* *}$ & $-0,05$ \\
5. Calidad Total & & & & & 1,00 & $-0,08$ \\
6. Grado de estudios & & & & & & 1,00 \\
\hline
\end{tabular}

Tabla $N^{\circ}$ 5: Correlaciones entre rendimiento académico y percepción de la calidad en primero de secundaria.

\begin{tabular}{lccccc}
\hline Nota & 1 & 2 & 3 & 4 & 5 \\
\hline 1. Nota promedio & 1,00 & $0,30^{*}$ & $0,43^{*}$ & $0,29^{*}$ & $0,37^{* *}$ \\
2. Calidad dirección & & 1,00 & $0,89^{* *}$ & $0,80^{* *}$ & $0,95^{* *}$ \\
3. Calidad docencia & & & 1,00 & $0,69^{* *}$ & $0,93^{* *}$ \\
4. Calidad administrativa & & & & 1,00 & $0,87^{* *}$ \\
5. Calidad Total & & & & & 1,00 \\
\hline
\end{tabular}

Tabla $N^{\circ}$ 6: Correlaciones entre rendimiento académico y percepción de la calidad en segundo de secundaria.

\begin{tabular}{lccccc}
\hline \multicolumn{1}{c}{ Nota } & 1 & 2 & 3 & 4 & 5 \\
\hline 1. Nota promedio & 1,00 & 0,18 & $0,23^{*}$ & 0,10 & $0,20^{* *}$ \\
2. Calidad dirección & & 1,00 & $0,67^{* *}$ & $0,42^{* *}$ & $0,88^{* *}$ \\
3. Calidad docencia & & & 1,00 & $0,55^{* *}$ & $0,86^{* *}$ \\
4. Calidad administrativa & & & & 1,00 & $0,74^{* *}$ \\
5. Calidad Total & & & & & 1,00 \\
\hline
\end{tabular}


Tabla $N^{\circ}$ 7: Correlaciones entre rendimiento académico y percepción de la calidad en tercero de secundaria.

\begin{tabular}{lccccc}
\hline Nota & 1 & 2 & 3 & 4 & 5 \\
\hline 1. Nota promedio & 1,00 & $-0,54^{* *}$ & $-0,45^{*}$ & $-0,39^{* *}$ & $-0,49^{* *}$ \\
2. Calidad dirección & & 1,00 & $0,86^{* *}$ & $0,89^{* *}$ & $0,97^{* *}$ \\
3. Calidad docencia & & & 1,00 & $0,79^{* *}$ & $0,93^{* *}$ \\
4. Calidad administrativa & & & & 1,00 & $0,93^{* *}$ \\
5. Calidad Total & & & & & 1,00 \\
\hline
\end{tabular}

Tabla $N^{\circ}$ 8: Correlaciones entre rendimiento académico y percepción de la calidad en cuarto de secundaria.

\begin{tabular}{lccccc}
\hline Nota & 1 & 2 & 3 & 4 & 5 \\
\hline 1. Nota promedio & 1,00 & $0,40^{* *}$ & $0,13^{*}$ & $-0,34^{* *}$ & $-0,36^{* *}$ \\
2. Calidad dirección & & 1,00 & $0,60^{* *}$ & $0,65^{* *}$ & $0,89^{* *}$ \\
3. Calidad docencia & & & 1,00 & $0,57^{* *}$ & $0,82^{* *}$ \\
4. Calidad administrativa & & & & 1,00 & $0,85^{* *}$ \\
5. Calidad Total & & & & & 1,00 \\
\hline
\end{tabular}

Tabla $N^{\circ}$ 9: Correlaciones entre rendimiento académico y percepción de la calidad en quinto de secundaria.

\begin{tabular}{lccccc}
\hline Nota & 1 & 2 & 3 & 4 & 5 \\
\hline 1. Nota promedio & 1,00 & $-0,17$ & $-0,18^{*}$ & $-0,30^{* *}$ & $-0,25^{* *}$ \\
2. Calidad dirección & & 1,00 & $0,61^{* *}$ & $0,57^{* *}$ & $0,89^{* *}$ \\
3. Calidad docencia & & & 1,00 & $0,60^{* *}$ & $0,84^{* *}$ \\
4. Calidad administrativa & & & & 1,00 & $0,82^{* *}$ \\
5. Calidad Total & & & & & 1,00 \\
\hline
\end{tabular}

psicométricas del instrumento.

Pero también se evidencia que no hay relaciones significativas entre las variables de estudio, vale decir entre el rendimiento académico de las estudiantes y la percepción de la calidad educativa. Sin embargo, al hacer las correlaciones por grado de escolaridad, sí se observa relaciones fuertes y significativas en casi todos los casos.

Por ejemplo, en primero de secundaria, se encontraron relaciones significativas entre todos los datos. Esto quiere decir que en este grado, el rendimiento académico se relaciona con la percepción de la calidad educativa total, a nivel de dirección, docentes y de los administrativos, dado que la correlación más baja fue de $r=$ 0,29 y la más alta de $r=0,95$ con un nivel de significancia de $p<0,05$ y $p<0,01$, respectivamente.

En el segundo de secundaria, las relaciones entre el rendimiento académico y la percepción de la calidad educativa, se dieron solo para la percepción de la calidad docente y la percepción de la calidad total. Estas relaciones fueron bajas pero significativas a un $p<0,05$. Las demás relaciones reflejan que los componentes del instrumento se relacionan entre sí de manera alta y significativa, con valores de $r=0,42$ a $r=0,88$, siendo mayores los puntajes entre la percepción de la calidad total y los tres factores que le componen (tabla 6).

En tercero de secundaria, todas las relaciones entre el rendimiento académico 
y la percepción de la calidad educativa fueron moderadas y significativas, con la salvedad de que fueron negativas. Lo que sugiere que en este grado, al aumentar la percepción de la calidad educativa, el rendimiento académico disminuye. Las relaciones entre los componentes del instrumento fueron altos, positivos y muy significativos $(p<0,01)$ con valores de $r=$ 0,79 a $r=0,97$.

En cuarto de secundaria, ocurre igual que en segundo y primero. Las relaciones entre el rendimiento académico son positivas y moderadas a excepción de la relación entre el rendimiento académico y la percepción de la calidad de la docencia, donde se obtuvo una relación de $r=0,13$. Todas las demás relaciones fueron moderadas y altas con un valor mínimo de $r=0,34$ y un valor máximo de $r=0,89$.

Finalmente, en quinto de secundaria, solo hubo relaciones significativas entre el rendimiento académico y la percepción de la calidad en administrativos y de manera global. Estas correlaciones fueron bajas y negativas. Todas las demás correlaciones entre los factores del instrumento fueron moderadas $y$ altas con valores entre $r=$ 0,57 y $r=0,89$.

\section{DISCUSIÓN}

La percepción más allá de ser un fenómeno psicológico, fisiológico y sensorial, es un proceso activo del procesamiento de la información, que además de integrar elementos señoriales de la realidad, favorece su interpretación, sobre la base de la experiencia previa; de modo que determina nuestras cogniciones, actitudes y conductas, hacia diversos hechos (22).

La percepción como fenómeno sensorial ha sido estudiada desde hace muchos siglos atrás. Aristóteles por ejemplo en De Anima, formula explicaciones a este fenómeno y lo considera como parte del conocimiento sensitivo. Santo Tomás de Aquino también le concibe como un nivel básico de procesamiento que se conecta con niveles superiores como el conocimiento intelectivo. En la edad media, Alhazen hace la primera propuesta de que la anatomía del ojo está diseñada para trabajar con la reflexión de la luz. Ya en la edad moderna los filósofos europeos han dado diversas aproximaciones a este fenómeno psicológico como Descartes, Locke, Berkeley, Kant, etc. (23).

Sería en el siglo XIX que mediante estudios neurofisiológicos el sustrato físico de la percepción sería conocido. Además, ya en el siglo XX, el aporte de los psicólogos de la gestalt, dejaría sentadas las bases psicológicas de la percepción y sus implicancias en diversos procesos cognitivos, como la memoria, la solución de problemas, el pensamiento productivo, la toma de decisiones, etc. (24).

Más recientemente, la percepción se ha estudiado en vinculación con diversos temas. En la medida que se ha adoptado un enfoque de la percepción como "manera de ver el mundo" y no sólo como fenómeno sensorial. Así pues, la visión de la realidad puede tener injerencia sobre nuestra conducta en contextos educacionales. Por ejemplo, en un estudio de Torres, Lajo, Campos y Riveros (20), se encontró que la percepción que tienen los estudiantes del nivel académico de sus profesores, no determina su rendimiento académico.

Nuestros resultados apuntan en otra dirección, ya que sí se encontraron relaciones significativas entre las variables estudiadas, pero estas no se dieron cuando se procesó la información del total de la muestra, sino cuando se realizó el análisis de datos según el grado de estudios. Esto sugiere que entre las relaciones de la percepción de la calidad educativa y el rendimiento académico median, las características de los estudiantes, las cuales pueden tener ciertos patrones comunes cuando se toma como criterio el grado de escolaridad.

En todas las correlaciones efectuadas, los valores obtenidos para las relaciones entre los factores del Cuestionario de percepción de la calidad en la escuela y 
el total del instrumento, fueron positivas y altas o moderadas con un nivel de significancia de $p<0,01$; lo cual no hace más que corroborar los índices de validez y confiabilidad que se obtuvieron en un análisis psicométrico previo.

Por otro lado, las correlaciones en primer grado de secundaria son significativas en su totalidad. Mientras que en segundo grado las relaciones significativas se dieron entre el rendimiento académico y la percepción de la calidad de la docencia y el total de la puntuación obtenida a través del instrumento diseñado. Esto sugiere que en segundo de secundaria, la calidad de la docencia puede tener cierta influencia en el rendimiento académico.

En tercero de secundaria y quinto de secundaria, las relaciones entre la percepción de la calidad educativa y el rendimiento académico, fueron negativas, con un nivel de significancia aceptable en la mayoría de los casos. Es decir que mientras mayor es la percepción de la calidad educativa, menor es el rendimiento académico. Estos resultados, aunque parecen ser contradictorios, pueden estar reflejando el estrés académico, que deviene de la transición que se aprecia en estos grados, debido a que las exigencias académicas son particularmente diferentes. En tercero de secundaria por un lado, aumentan cursos de ciencias como química y física; mientras que en quinto de secundaria, las estudiantes tienen que decidir qué carrera estudiar en relación con su futuro laboral.

Es posible que en estos grados se manifiesten situaciones sui generis, aunque no se descarta, la presencia de otros factores, que hayan podido interferir en las correlaciones efectuadas, lo cual constituiría una limitación en nuestro estudio, al no haber ejercido el control suficiente sobre dichas variables. En ese sentido, la conducta rebelde durante la adolescencia (25), podría explicar los datos obtenidos, aunque eso supondría hacer valoraciones de esta variable. El sexo también podría tener injerencia en los resultados, ya que se trabajó con una muestra compuesta por mujeres en su totalidad.

En cuarto de secundaria las relaciones entre las variables de estudio, vuelven a ser positivas y significativas como pasa con las estudiantes de primero y segundo de secundaria. Con la salvedad de que la relación entre rendimiento académico y la percepción de la calidad en docencia, fue baja y no significativa.

Ahora bien, la realización de este estudio es un importante antecedente para la investigación de la calidad educativa en la región Arequipa, sobre la base de dos estudios previos realizados en la ciudad de Lima (20). No se puede soslayar la investigación en un campo tan relevante como el de la calidad educativa en el país y las provincias. A raíz de este trabajo de investigación, sugerimos que se amplíe la muestra y se analicen las relaciones entre otras variables ya que nuestro estudio no permite la generalización de los resultados hallados. Además, dado que el instrumento diseñado para este estudio, cuenta con altos índices de validez y también de confiabilidad, debería ser usado en estudios similares o bien para evaluar la calidad educativa al interior de los colegios de la región.

En ese sentido, nuestro estudio aporta de dos maneras al campo de la educación. En primer lugar, a través de la generación de conocimiento, cuyo valor teórico recae en los datos hallados con respecto a las relaciones entre las variables de estudio: el rendimiento académico y la percepción de la calidad educativa. En segundo lugar, el valor metodológico del estudio reposa en la construcción de un instrumento que permite valorar objetiva y cuantitativamente, la percepción de la calidad educativa, que bien puede ser utilizado con fines de investigación o con fines prácticos en las diversas instituciones educativas.

Tras haber hecho el procesamiento de la información y el respectivo análisis de datos, se concluye que nuestra hipótesis se cumple de forma parcial, ya que si bien, 
en el total de la muestra no ha habido relaciones significativas entre la percepción de la calidad educativa y el rendimiento académico, sí se han encontrados diversas relaciones válidas y significativas entre estas variables, según el grado de estudios de las alumnas de la IE "Arequipa". Esto sugiere que en la percepción de la calidad educativa, el grado de escolaridad es un factor importante.

\section{REFERENCIAS BIBLIOGRÁFICAS}

1. Ishikawa K. Control total de la calidad. Valparaíso: Alfa S.A; 1994.

2. Álvarez GV. El aprendizaje organizacional como eje de desarrollo en la organización. Educación. 2006; 15(29): 7-34.

3. Salazar M, García J. Calidad total. Lima: CINSEYT; 1996.

4. Capella J. Gestión de la calidad en la institución educativa. Educación. 2006; 15(28): $21-59$.

5. Vásquez KB. Los profesores directores que laboran en escuelas unidocentes del ámbito rural y sus necesidades de capacitación en gestión. Educación. 2007; 16(31): 63-80.

6. Huaranga $O$. Proyecto de desarrollo institucional. Lima: San Marcos; 2001.

7. Contreras BM. Liderazgo directivo en la gestión escolar desde el enfoque político de la escuela. Educación. 2009; 18(34): 55-72.

8. Revilla DM. Modelo de autoevaluación institucional global con apoyo externo de un centro educativo particular de Lima. Educación. 2008; 17(33): 63-80.

9. Huaranga $O$. Estrategia de proyectos. Lima: San Marcos; 1999.

10. Sovero FV. Innovadores planes y proyectos educativos. Lima: San Marcos; 2003.

11. Rodríguez CP El programa Nacional de Formación y Capacitación Permanente (PRONAFCAP). Educación. 2010; 19(37): 87-103.

12. Huaranga $O$. Calidad educativa $y$ enfoques constructivistas. Lima: San
Marcos; 2000.

13. Vásquez MI. La autogestión de procesos de cambio en centros educativos. Educación. 2013; 22(42): 117-134.

14. Drucker P El management del futuro. Buenos Aires: Sudamericana; 2003.

15. Miranda JC. Una referencia particular sobre el concepto de calidad de la educación. Zona próxima. 2006; 7: 132-145.

16. Filho L. Organización y administración escolar. Argentina: Kapeluz; 1979.

17. Montes I. Estándares para mejorar la calidad de nuestra educación. En Alfaro L. TIC's en Educación, p. 271-302. Arequipa: UNSA; 2005.

18. Arévalo RM. Teorías de dominio de los docentes sobre el aprendizaje y su expresión en la evaluación de los aprendizajes: Un estudio de caso en una institución educativa particular de Lima. Educación. 2010; 19(36): 23-42.

19. Yamada G, Castro F. Dos propuestas concretas para mejorar la calidad y equidad de la educación peruana. Lima: Universidad del Pacífico; 2011.

20. Torres M, Lajo R, Campos E, Riveros $M$. Rendimiento académico de los alumnos de una facultad de educación de una universidad pública de Lima y su percepción de la calidad académica de los docentes. Revista de Investigación en Psicología. 2007; 10(1): 71-89.

21. Hernández R, Fernández C, Baptista P Metodología de la investigación. México: McGraw-Hill; 2006.

22. Arias WL. Fundamentos del aprendizaje. Arequipa: Vicarte; 2008.

23. Hothersall D. Historia de la psicología. México: McGraw-Hill; 1997.

24. Whittaker JO. Psicología. México: Interamericana; 1993.

25. Arias WL. Agresión y violencia en la adolescencia: La importancia de la familia. Avances en Psicología. 2013; 21(1): 23-34. 